\title{
Precision of Taylor Dispersion
}

\author{
Patricia Taladriz-Blanco, ${ }^{\dagger}$ Barbara Rothen-Rutishauser, ${ }^{\dagger}$ Alke Petri-Fink, ${ }^{\dagger}+\odot$ and Sandor Balog* ${ }^{*}{ }^{\dagger}$ \\ ${ }^{\dagger}$ Adolphe Merkle Institute, University of Fribourg, Chemin des Verdiers 4, 1700 Fribourg, Switzerland \\ ${ }^{\ddagger}$ Chemistry Department, University of Fribourg, Chemin du Musée 9, 1700 Fribourg, Switzerland
}

Supporting Information

\begin{abstract}
Taylor dispersion is capable of measuring accurately the hydrodynamic radius over several orders of magnitude. Accordingly, it is now a highly competitive technique dedicated to characterizing small molecules, proteins, macromolecules, nanoparticles, and their selfassembly. Regardless, an in-depth analysis addressing the precision of the technique, being a key indicator of reproducibility, is not available. Benefiting from analytical modeling and statistical analysis, we address error propagation and present a comprehensive theoretical study of the precision of Taylor dispersion. Theory is then compared against experiment, and we find full consistency. Our results are

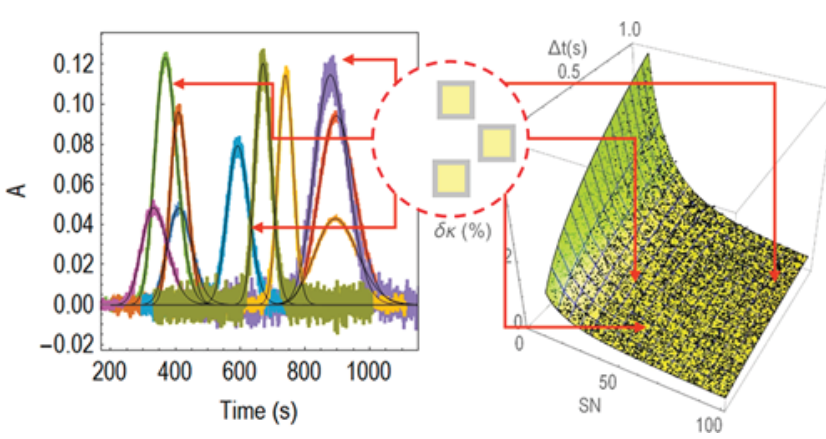
most helpful when the design, objectives, or control of analytical quality is in focus.
\end{abstract}

S imilar to dynamic light scattering, Taylor dispersion gives access to measuring the hydrodynamic radius while being considerably less sensitive to the moderate presence of impurities. ${ }^{1,2}$ Therefore, there is considerable interest shown by the fields of material science, pharmacology and toxicology. ${ }^{3-24}$

The technique combines three physical phenomena: optical extinction, translational self-diffusion, and sheer-enhanced dispersion created by a steady laminar flow. ${ }^{25-27}$ The velocity profile of the laminar flow, which is usually driven by a pressure gradient in a microfluidic channel, disperses the initially narrow band of the injected analyte and creates a concentration gradient that induces a spontaneous net transport of particles via translational self-diffusion. As a result, the band broadens, and the rate of band-broadening is defined by the flow profile and translational diffusion coefficient of the particles.

The taylorgram is the temporal record of the optical absorbance of the particle band at the detection point. Given the Lambert-Beer law and a linear detector response, the taylorgram of uniform particles of hydrodynamic radius $r$ resulting from an experiment where the volume of the injected sample band is small and dispersed in a cylindrical channel, and the detection is point-like and is written as follows: ${ }^{25-27}$

$$
A(t)=A_{0} \frac{1}{\sqrt{\pi \cdot \kappa \cdot t}} \mathrm{e}^{-\left(t-t_{0}\right)^{2} / \kappa \cdot t}
$$

where $A_{0}$ is the amplitude, $\kappa=r \pi \eta Y^{2} /\left(2 k_{\mathrm{B}} T\right), Y$ is the capillary radius, $T$ is the temperature, $\eta$ is the viscosity of the fluid, $k_{\mathrm{B}}$ is the Boltzmann constant, and $t_{0}=x / v$ is the so-called residence time defined by the distance between detection and injection points and the mean velocity of the flow $(v)$. The temporal mean and variance of the absorbance profile is respectively $t_{0}+$

$\kappa / 2$ and $\left(t_{0}+\kappa\right) \kappa / 2$. When the residence time $t_{0}$ is much larger than $\kappa$, eq 1 is essentially a Gaussian curve, and one can accurately measure the hydrodynamic radius by determining $\kappa$ from the absorbance profile

$$
r=\frac{2 k_{\mathrm{B}} T}{\pi \eta Y^{2}} \kappa
$$

Taylor dispersion, as any experimental technique, is subject to errors. The impact of random errors and the consequent uncertainty, which is usually referred to as precision, is a key indicator of measurement quality. The concept of precision, quantified either as standard deviation $(\Delta r)$ or relative standard deviation $(\Delta r /\langle r\rangle)$ of a set of measurements, is generally considered at three levels: (1) repeatability, (2) intermediate precision, and (3) reproducibility. Repeatability, also referred to as either intra-assay or within-run precision, addresses the precision obtained with identical operating conditions over a short interval of time. This level of precision typically describes data obtained by the same operator in a single run composed of a series of measurements with the same instrument and same operational settings within a given laboratory. Intermediate precision expresses variations obtained on different days by different operators with different settings using the same instrument within a given laboratory. Reproducibility expresses the precision between laboratories and instruments, typically, in collaborative studies established for standardization of methodologies. Therefore, while the uncertainty of some parameters, for example, the ones 
describing fluid viscosity as a function of temperature, may be invariant within these three levels, other parameters, such as the capillary radius, may vary within these levels, that is, between instruments and different laboratories.

Accordingly, reliable information regarding the expected uncertainty is crucial. Perhaps the most relevant example is the approach of analytical quality by design (AQbD), where the analytical target profile (ATP) defines the objectives and criteria of the reportable result, such as the precision necessary to adequately characterize the critical quality attributes (CQA) of a given process. ${ }^{28-31}$ Notably, in pharmaceutical research, these metrics represent a serious economic factor, ${ }^{28}$ and the related scientific and regulatory knowledge requires stringent analytical standards. ${ }^{32}$

Despite the merits, an in-depth analysis addressing the precision of Taylor dispersion is not available, which defines the motivation as well as the subject of our paper. Our aim is to describe quantitatively the factors that affect the precision of Taylor dispersion, and our focus is on analytical modeling and statistical analysis of error propagation. Finally, to test our model, the theoretical calculations will be compared against experimental analyses.

According to eq 2, there are four basic variables that are directly relevant to the precision of Taylor dispersion: (1) capillary radius $Y,(2)$ width-parameter of the taylorgram $\kappa,(3)$ temperature $T$, and (4) viscosity $\eta$. To describe precision, we will use the formalism of propagation of small errors. ${ }^{33}$ In this formalism, the precision of measuring $r$ is independent of the exact shapes of the density distributions of the corresponding variables, but is a function of their degree of uncertainty, that is, their standard deviations. Our choice is deliberate and justified because in Taylor dispersion experiments the uncertainties of the corresponding variables are indeed small. Nonetheless, this choice does not imply that the treatment of arbitrary large errors is not feasible. Arbitrary large errors may be addressed through the full shape of the density distributions and the Jacobian matrix of partial derivatives of the corresponding variables. ${ }^{34}$

The uncertainty regarding the capillary radius can be estimated via the tolerance value $\left(Y_{\text {tol }}\right)$, which is usually provided by the manufacturer. In most cases, the tolerance value is symmetric and represents upper and lower limits bounding the expected uniform variation around the nominal value. Accordingly, the corresponding uncertainty is the standard deviation of a uniform distribution: $\Delta Y=1 / \sqrt{ } 3 Y_{\text {tol }}$. To illustrate the calculation of propagation of arbitrary large errors, we rely on the premises that (a) the analytic function relating $r$ to $Y$ is known and (b) the distribution function of $Y$ is also known. The marginal distribution function of $r$ can be obtained by applying the rule of transforming of random variables. Therefore, while the distribution of $Y$ is uniform, it is not difficult to show that, due to the nonlinear relationship, the distribution of $r$ is neither uniform nor symmetric. The probability density scales as $p(r) \propto r^{-3 / 2}$ on the closed interval defined by the tolerance value. This means that, in the case of uncertainty in $Y$, the most probable value of $r$ is shifted to the lowest possible value set by the given tolerance of $Y$. This systematic error is, however, usually small, typically less than $0.1 \%$, and not relevant in the practice of Taylor dispersion. Owing to thermal expansion, the capillary radius is also a function of temperature, but given that the linear thermal expansion coefficient of fused silica (which is the most frequently used capillary material used in Taylor dispersion) is very small (approximately $10^{-6}{ }^{\circ} \mathrm{C}^{-1}$ ), here we will not pay attention to this effect.

We expect that the properties of the taylorgram itself play a role in the uncertainty of determining $\kappa$. Accordingly, the magnitude of $\Delta \kappa$ is a function of the properties of the sample itself, that is, the concentration, optical extinction, and hydrodynamic radius of the particles. We also anticipate that $\Delta \kappa$ is a function of the parameters of the experiment, such as residence time $\left(t_{0}\right)$, temporal resolution $(\tau)$, and signal-tonoise ratio $(S N)$. To understand the roles these factors play, first we consider what defines the quality of a taylorgram.

Owing to their quantized nature, detecting photons is intrinsically random, and the consequence is that the number of photons detected during $\tau$ time is a random variable. When one measures the absorbance three times under exactly the same conditions, one is likely to obtain three different results, even if the intensity of the illumination is completely stable. This is because the probability density function of the photon counts will follow a Poisson distribution. ${ }^{35}$ This randomness and the consequential uncertainty is not resulting from faulty measurements: it is an inherent property of classical linear spectroscopy and referred to as shot noise. Accordingly, measuring optical transmission $(T)$ and absorbance $(A=$ $\left.-\log _{10}(T)\right)$ is always uncertain. The detailed discussion is presented in the Supporting Information (The signal-to-noise ratio of taylorgrams).

Additionally, Taylor dispersion is a linear time-invariant system, and a small temporal resolution and a large detection area result in a convoluted expression of eq 1 , which results in a notable systematic error unless a dedicated approach is taken in the analysis. ${ }^{2}$ Therefore, the quality of the taylorgram is dependent on the sample properties, such as the hydrodynamic radius defining the value of $\kappa$, the optical extinction, and the concentration of the particles. The signal-to-noise ratio is higher when the optical extinction and concentration of the particles are high because, in this case, the optical transmission is smaller. The residence time $\left(t_{0}\right)$ and $\kappa$ will define the minimum temporal resolution $(\tau)$ necessary to adequately resolve the center and width of the taylorgram. When $\tau$ is, however, too small, the signal-to-noise ratio may be insufficiently low. Therefore, there is a trade-off to seek in resolution and noise level.

Being inspired by Buckingham's theorem, ${ }^{36}$ we propose that the relative uncertainty of determining $\kappa$ is expressed as a power function of four parameters

$$
\frac{\Delta \kappa}{\kappa}=z \kappa^{B} t_{0}{ }^{C} \tau^{D} S N^{E}
$$

where $z$ is a dimensionless constant, $\kappa$ is the value defined by the uniform hydrodynamic radius of the particles, $t_{0}$ is the residence time, $\tau$ is the time resolution recording the taylorgram, and $S N$ is the signal-to-noise ratio, which is also dimensionless. Given that the units of $\kappa, t_{0}$, and $\tau$ are identical $(s)$, the dimensionality of eq 3 requires that $B+C+D=0$. We can express similarly the relative error of determining the residence time $\Delta t_{0} / t_{0}$, which is necessary when the combination of two-window analyses is used. ${ }^{12}$

To determine the parameters in eq 3 , we simulated and analyzed 100000 fully realistic taylorgrams. All details are given in the Supporting Information (The uncertainty of determining the width and the center of a taylorgram). Table 1 lists the parameters of eq 3 obtained in these simulated experiments. 
Table 1. Parameters of Eq 3 Describing the Relative Error of Determining the Width and the Center of Fully Realistic Taylorgrams of Uniform Particles

\begin{tabular}{lclccc} 
& $z$ & $B(\kappa)$ & $C\left(t_{0}\right)$ & $D(\tau)$ & $E(S N)$ \\
$\Delta \kappa / \kappa$ & 2.42 & -0.24 & -0.26 & 0.50 & -1 \\
$\Delta t_{0} / t_{0}$ & 0.85 & 0.26 & -0.76 & 0.50 & -1 \\
\hline
\end{tabular}

To summarize, the uncertainty of both $\kappa$ and $t_{0}$ scales with $\sqrt{ } \tau / S N$. The expected precision of measuring $\kappa$ increases with both hydrodynamic radius and residence time, and the expected precision of measuring $t_{0}$ is better at small hydrodynamic radius. The precision of determining $t_{0}$ is in general better than that of $\kappa$.

Temperature and viscosity are not independent variables, for the viscosity is a function of temperature: $\eta=f(T)$. Therefore, in most Taylor dispersion experiments the actual viscosity is not measured directly, but calculated from the nominal system temperature set for the experiment. According to the specifications of typical electrophoresis injection systems, temperature is measured and controlled with an uncertainty not larger than $T_{\text {tol }}= \pm 0.5 \mathrm{~K}$. Therefore, in this view, the actual temperature value lies within a uniform distribution whose standard deviation is $\Delta T=1 / \sqrt{ } 3 T_{\text {tol }}$.

Viscosity can also be measured directly in Taylor dispersion experiments by combining the analyses of two detection points and applying the Hagen-Poiseuille equation. ${ }^{16}$ By determining the centers of the two taylorgrams obtained from the same injection event, the mean flow velocity can be determined via the ratio

$$
V=\frac{L_{2}-L_{1}}{t_{2}-t_{1}}
$$

where the dispersion is recorded at two detection windows: $t_{1}$ and $t_{2}$ are the residence times and $L_{1}$ and $L_{2}$ are the distances between injection and the two detection points. The fluid viscosity then can be expressed as

$$
\eta=\frac{Y^{2} P}{8 L V}
$$

where $P$ is the driving pressure, $L$ is the capillary length, and $Y$ is the capillary radius. In this way, the actual sample temperature can be evaluated from the actual viscosity by using the inverse function defining the viscosity-temperature relationship: $T=f^{-1}(\eta)$.

The method of using temperature and viscosity will also define how the related uncertainty propagates and how it must be addressed. This is because, in the first case, $r$ is determined via $T / f(T)$, while in the second case via $f^{-1}(\eta) / \eta$. We address both scenarios here and compare them with our experimental results.

For the experiments, taylorgrams of a dilute aqueous dispersion $(0.5 \mathrm{~g} / \mathrm{L})$ of bovine serum albumin (BSA) were collected (Figure 1). In our experiments, the duration of the pressure ramp ( $3 \mathrm{~s}$ ) was negligible compared to the residence times, and each aliquot volume was estimated as $2.2 \%$ and $1 \%$ compared to the capillary volume until the first and second window, respectively. Accordingly, we did not apply correction, and the hydrodynamic radius determined at the second window via eq 2 is expected to be highly accurate. ${ }^{12,37}$ All the details are presented in the Supporting Information (Taylor dispersion experiments of BSA).
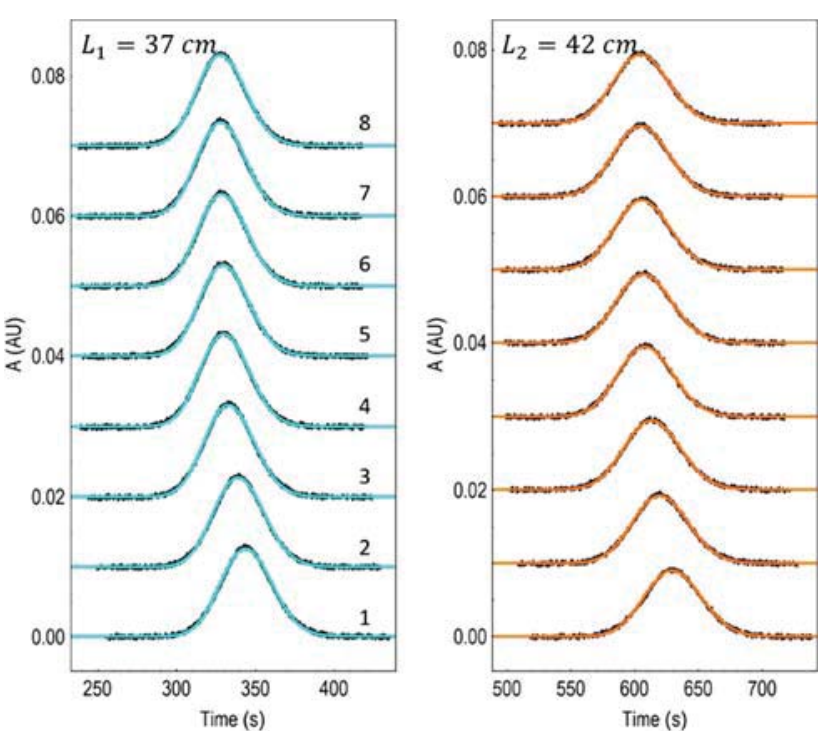

Figure 1. Eight pairs of taylorgrams (in black) recorded in a single run and the corresponding best fits of eq 1 (in color). These fits determine the centers (residence times: $t_{1}$ and $t_{2}$ ) and widthparameters $(\kappa)$ of the taylorgrams. Profiles are shifted vertically for the sake of clarity.

To fit eq 1 against the experimental data, the unconstrained nonlinear model fit was used (Mathematica, Wolfram Language, Wolfram Research, Inc., version 11.3, Champaign, IL). The taylograms and the corresponding best fits are shown in Figure 1. All of the fit parameters obtained are listed in the Supporting Information (Model parameters).

During recording the taylorgrams the aliquots were sampled from the same dispersion, and a single set of instrument parameters were used. This set included constant capillary diameter $(Y)$, capillary length $(L)$, injection-to-detection distances $\left(L_{1}, L_{2}\right)$, and temporal resolution $(\tau)$. Therefore, in these experiments there were no random variabilities describing these parameters, that is, $\Delta Y=\Delta L=\Delta L_{1}=\Delta L_{2}$ $=\Delta \tau=0$.

Random errors concerned pressure $(P)$, residence times $\left(t_{1}\right.$, $\left.t_{2}\right)$, width parameter $(\kappa)$, signal-to-noise ratio $(S N)$, temperature $(T)$, and viscosity $(\eta)$.

To describe the viscosity of water as a function of the temperature (Supporting Information; The viscosity of water as a function of temperature, Figure SI 4), we used an exponential expression with three free parameters: ${ }^{38,39}$

$$
f(T)=a e^{b /(T-c)}
$$

The parameters of eq 6 were kept constant in all the calculations and, therefore, showed no random variabilities, that is, $\Delta a=\Delta b=\Delta c=0$. Accordingly, the expectable uncertainty resulting from our experiments, expressed as the relative standard deviation, can be written as

$$
\frac{\Delta r}{r}=\sqrt{\left(\frac{\Delta T}{T}\right)^{2}+\left(\frac{\Delta \eta}{\eta}\right)^{2}+\left(\frac{\Delta \kappa}{\kappa}\right)^{2}}
$$

The error-propagation term addressing the capillary radius $2 \Delta Y / Y$ is not included in eq 7 , because for all the measurements we presented and analyzed, one capillary was used, and consequently, this term vanishes in our experiment. This term is relevant when results are obtained with different 
capillaries that are, for example, nominally identical (such as, produced by the same manufacturer, the same brand, type, tolerance, etc.). Using different capillaries would be typical for inter- and intralaboratory campaigns. The uncertainty regarding the capillary radius can be estimated via the tolerance value $\left(Y_{\text {tol }}\right)$, which is usually provided by the manufacturer and generally changes with manufacturer, brand, type, and inner radius. In most cases, the tolerance value is symmetric and represents an upper-lower limit bounding the expectable uniform variation around the nominal value. Accordingly, the corresponding uncertainty is the standard deviation of a uniform distribution: $\Delta Y=1 / \sqrt{ } 3 Y_{\text {tol }}$, and the uncertainty originating from the capillary radius may easily reach $5 \%$.

In our experiments, $T$ was set to $298.15 \mathrm{~K}$. According to the specifications of our capillary electrophoresis injection system, $\Delta T$ was expected to be $0.29 \mathrm{~K}$. When viscosity is not measured directly, but evaluated via the nominal temperature set in the experiment, the expected relative error is

$$
\frac{\Delta \eta}{\eta}=\frac{b}{(c-T)^{2}} \Delta T
$$

When viscosity is measured directly (eqs 4 and 5), the actual temperature of the experiment is determined through the viscosity via the inverse of eq 6 (Supporting Information, The viscosity of water as a function of temperature, Figure SI 4). Our results show that, despite the fact that the temperature was set constant in our experiment, it first increased and then saturated (Figure 2).
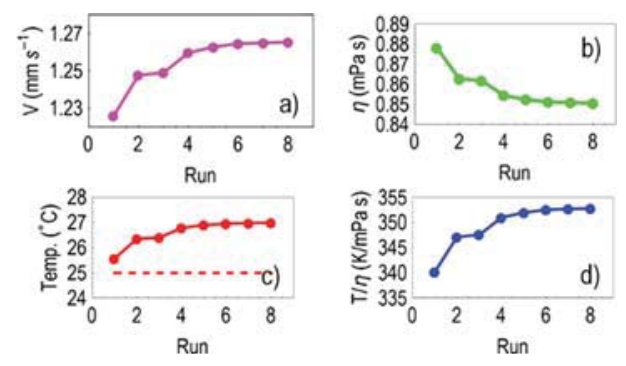

Figure 2. Parameters obtained from the analysis of the centers of the taylorgrams. Panels display (a) flow velocity, (b) viscosity, (c) temperature, where the dashed line indicates the nominal temperature set, and (d) the temperature-viscosity ratio.

This means that, when the temperature and viscosity were taken as constants, a systematic error of $5 \%$ affected accuracy. A continuously increasing temperature profile is a plausible outcome of light absorption and consequent heat dissipation changing the temperature of the running fluid. Indeed, a straightforward mathematical model describing uninsulated heating is able to capture accurately the profile Figure 3 features (Supporting Information, Capillary flow temperature by light absorption and heat dissipation).

Accordingly, the viscosity decreased and the velocity of the laminar flow increased from measurement to measurement. The relative error describing the certainty of viscosity is

$$
\frac{\Delta \eta}{\eta}=\sqrt{\left(\frac{\Delta P}{P}\right)^{2}+\left(\frac{\Delta t_{1}}{t_{1}-t_{2}}\right)^{2}+\left(\frac{\Delta t_{2}}{t_{1}-t_{2}}\right)^{2}}
$$

where according to the manufacturer the relative error of the driving pressure $(\Delta P / P)$ was $1 \%$. The relative error describing the certainty of temperature is

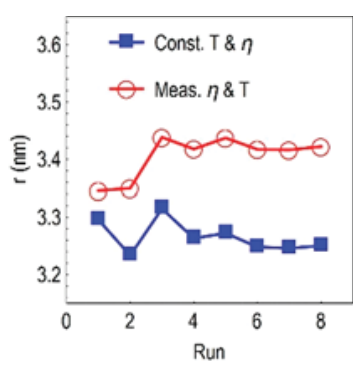

Figure 3. Hydrodynamic radius of BSA obtained in our experiments, determined at the second window. In the first case (blue), the temperature and viscosity were taken as constants $\left(25^{\circ} \mathrm{C}\right)$ set by the instrument. In the second case (red), the viscosity was measured through the Hagen-Poiseuille equation, and the temperature was determined via $f^{-1}(\eta)$ (eq 6).

$$
\frac{\Delta T}{T}=\frac{b}{b \ln (\eta / a)+c \ln (\eta / a)^{2}} \frac{\Delta \eta}{\eta}
$$

Now that we have all the necessary formulas and parameters to predict the precision, we can evaluate them and compare the results with our experiments. The signal-to-noise ratio, residence time, width parameter, and corresponding errors were estimated from the taylorgrams and model parameters as presented above (Supporting Information, The signal-to-noise ratio of taylorgrams, Model parameters).

The eight instances of the hydrodynamic radii determined at the second window are shown in Figure 3. Owing to the fact that the actual temperature values were systematically off, by using the nominal constant temperature and viscosity, we determine radii smaller than the ones obtained by using accurate viscosity and temperature. The inaccuracy is not critical, but increases gradually from 0.05 to $0.17 \mathrm{~nm}$. The mean of the correct values is $\langle r\rangle=3.41 \mathrm{~nm}$, which is in perfect agreement with the values reported in the literature. ${ }^{20,40-45}$ The relative standard deviation $\Delta r /\langle r\rangle$ is $1.08 \%$. The value predicted by our uncertainty model (eqs $6,7,9$, and 10) is $1.02 \%$. The agreement of the theoretical prediction with the experiment is very good. Nonetheless, the radius exhibits a jump between the second and the third runs. We attribute this to the fact that our instrument was not able to maintain the temperature we set for the runs, and the temperature drift resulted in a small yet systematic drift in the precision as well. Equation 7 shows that the precision is temperature-dependent, and accordingly, the runs are not truly homoscedastic from a strict mathematical point of view. Apart from this systematic experimental error that we were unable to avoid, these values still fit very well with the predicted theorical distributions. To show this, we amplified the volume of the extracted information by resampling and creating distinct $n$-subsets of the original set of the eight hydrodynamic radii. A single $n$ subset contains exactly $n$ elements of the original set, and the total number of different $n$-subsets is given by the binomial coefficient: $N=\left(\begin{array}{l}8 \\ n\end{array}\right)$. For example, there were 70 subsets that contained exactly four elements. The experimental sample statistic, that is, the relative standard deviation of the $N n$ subsets, is then compared with the statistic of the corresponding sampling distributions predicted by theory.

The theoretical sampling distribution of the relative standard deviation of Gaussian variables is presented in the Supporting Information (Sampling distributions of normally distributed random variables). Figure 4 displays the result obtained on the 
subsets of $n=2,3, \ldots, 7$. Again, experiment and theory show outstanding agreement with one another.
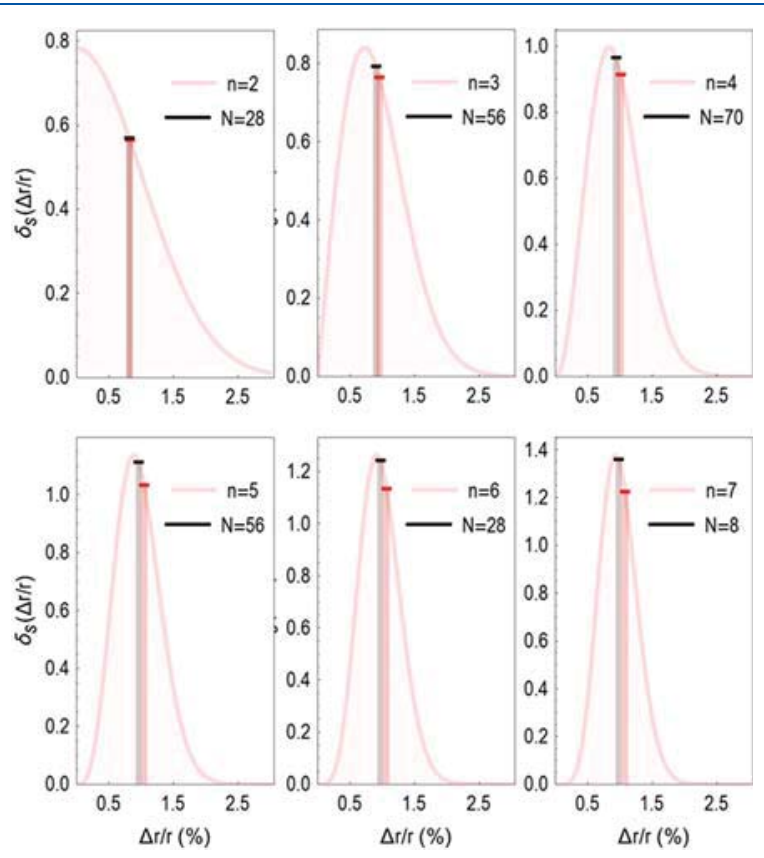

Figure 4. Theoretical sampling distributions of the relative standard deviation corresponding to different sample sizes. The vertical lines mark the positions of the experimental sample mean (black) and the mean of the theoretical sampling distribution (light red). The labels indicate the sample size $(n)$ and the total number of the corresponding subsets $(N)$.

To summarize, using theoretical modeling and statistical analysis, we studied exhaustively the precision of Taylor dispersion. The ability to determine accurately the expected precisions is most helpful when either the design or objectives or control of analytical quality is of importance. The general theoretical scenario was narrowed down to address the specific case of within-run precision, and the agreement with experimental results was excellent.

To conclude, Taylor dispersion is able to offer a high degree of reproducibility if the factors critical to quality are kept at bay. Notably, the relative error resulting from the capillary radius alone may exceed 5\%, and given that the relationships describing temperature and fluid viscosity are dominantly phenomenological, the calibration curve with the corresponding mathematical model itself is not free from uncertainty, and thus, the total relative error may surpass $3 \%$. (In this study, we used an aqueous dispersion, but evidently, water is not the only solvent suitable for error propagation analysis.) The least problematic factor appeared to be the analysis of the taylogram itself, for the corresponding relative error may arrive well below $1 \%$. The reason for this is the fact that optical absorbance with high signal-to-noise ratio and high temporal resolution can be readily obtained by using commercially available lightdetection solutions.

It is a frequent approach that the hydrodynamic radius is also measured by the combination of two windows, for example, when the injection volume is large enough compared to the "until-the-window" capillary volume. ${ }^{12,20}$ In this case, the accuracy may be improved, but the precision will decrease because additional independent variables are included. The formula of determining the precision in this way is presented in the Supporting Information (Two-window combination of determining the hydrodynamic radius).

Furthermore, by adapting the concept of statistical moments, the temporal moments (mean and variance) are frequently used to characterize multimodal and polydisperse samples and their optical extinction-weighted average radius. ${ }^{6,12,20,23,46,47}$ In this case, noise affects the attainable precision differently than it affects model-based nonlinear regression, and it can be shown that the precision of modelbased fit is superior to the precision obtained via temporal moments, independently on the multimodality and polydispersity of the samples studied (Supporting Information, Impact of noise on numerical integration and temporal moments).

Finally, using the concepts of inferential statistics to design an experiment with the desired level of quality is crucial. When one determines the precision via the propagation of errors, the uncertainties of the relevant parameters describe the population, which is by definition is the ensemble of all the attainable measurements. Therefore, the sampling distribution of the corresponding sample statistic (e.g., relative standard deviation) should be used when determining the number of measurements necessary to keep the sample-to-sample variations within desired limits.

\section{ASSOCIATED CONTENT}

\section{Supporting Information}

The Supporting Information is available

Figures, parameters, and mathematical relationships relevant to the findings of the manuscript (PDF)

\section{AUTHOR INFORMATION}

\section{Corresponding Author}

*E-mail: sandor.balog@unifr.ch.

\section{ORCID}

Patricia Taladriz-Blanco: 0000-0002-2469-9704

Alke Petri-Fink: 0000-0003-3952-7849

Sandor Balog: 0000-0002-4847-9845

\section{Author Contributions}

P.B.T. prepared the sample, operated the instrument, and collected the taylorgrams. B.R.R. and A.P.F. supervised P.B.T. S.B. conceived the idea, designed and analyzed the experiments, and wrote the manuscript through contributions of the coauthors.

\section{Notes}

The authors declare no competing financial interest.

\section{ACKNOWLEDGMENTS}

The authors are grateful for the financial support of the Adolphe Merkle Foundation, the University of Fribourg, and the Swiss National Science Foundation through the National Centre of Competence in Research Bio-Inspired Materials.

\section{REFERENCES}

(1) Hawe, A.; Hulse, W. L.; Jiskoot, W.; Forbes, R. T. Pharm. Res. 2011, 28, 2302-2310.

(2) Urban, D. A.; Milosevic, A. M.; Bossert, D.; Crippa, F.; Moore, T. L.; Geers, C.; Balog, S.; Rothen-Rutishauser, B.; Petri-Fink, A. Colloid and Interface Science Communications 2018, 22, 29-33.

(3) Bello, M. S.; Rezzonico, R.; Righetti, P. G. Science 1994, 266, 773-776. 
(4) Belongia, B. M.; Baygents, J. C. J. Colloid Interface Sci. 1997, 195, 19-31.

(5) Wuelfing, W. P.; Templeton, A. C.; Hicks, J. F.; Murray, R. W. Anal. Chem. 1999, 71, 4069-4074.

(6) Cottet, H.; Biron, J.-P.; Martin, M. Anal. Chem. 2007, 79, 90669073.

(7) d'Orlyé, F.; Varenne, A.; Gareil, P. Journal of Chromatography A 2008, 1204, 226-232.

(8) Le Saux, T.; Cottet, H. Anal. Chem. 2008, 80, 1829-1832.

(9) Medina, C.; Santos-Martinez, M. J.; Radomski, A.; Corrigan, O. I.; Radomski, M. W. Br. J. Pharmacol. 2007, 150, 552-558.

(10) Cottet, H.; et al. Anal. Chem. 2010, 82, 1793-1802.

(11) Hulse, W.; Forbes, R. Int. J. Pharm. 2011, 416, 394-397.

(12) Chamieh, J.; Oukacine, F.; Cottet, H. J. Chromatogr A 2012,

1235, 174-177.

(13) Cipelletti, L.; Biron, J.-P.; Martin, M.; Cottet, H. Anal. Chem. 2014, 86, 6471-6478.

(14) Jensen, S. S.; Jensen, H.; Cornett, C.; Møller, E. H.; Østergaard, J. J. Pharm. Biomed. Anal. 2014, 92, 203-210.

(15) Cipelletti, L.; Biron, J.-P.; Martin, M.; Cottet, H. Anal. Chem. 2015, 87, 8489-8496.

(16) Lavoisier, A.; Schlaeppi, J.-M. mAbs 2015, 7, 77-83.

(17) Oukacine, F.; Morel, A.; Desvignes, I.; Cottet, H. J. Chromatogr A 2015, 1426, 220-225.

(18) Pyell, U.; Jalil, A. H.; Urban, D. A.; Pfeiffer, C.; Pelaz, B.; Parak, W. J. J. Colloid Interface Sci. 2015, 457, 131-140.

(19) Høgstedt, U. B.; Schwach, G.; van de Weert, M.; Østergaard, J. Eur. J. Pharm. Sci. 2016, 93, 21-28.

(20) Balog, S.; Urban, D. A.; Milosevic, A. M.; Crippa, F.; RothenRutishauser, B.; Petri-Fink, A. J. Nanopart. Res. 2017, 19, 287.

(21) Fichtner, A.; Jalil, A.; Pyell, U. Langmuir 2017, 33, 2325-2339.

(22) Zaman, H.; Bright, A. G.; Adams, K.; Goodall, D. M.; Forbes,

R. T. Int. J. Pharm. 2017, 522, 98-109.

(23) Balog, S. Anal. Chem. 2018, 90, 4258-4262.

(24) Oukacine, F.; Gèze, A.; Choisnard, L.; Putaux, J.-L.; Stahl, J.-P.; Peyrin, E. Anal. Chem. 2018, 90, 2493-2500.

(25) Taylor, G. Proc. R. Soc. Lond. A 1953, 219, 186-203.

(26) Taylor, G. Proc. R. Soc. Lond. A 1954, 225, 473-477.

(27) Aris, R. Proc. R. Soc. Lond. A 1956, 235, 67-77.

(28) Rathore, A. S.; Winkle, H. Nat. Biotechnol. 2009, 27, 26.

(29) Vogt, F. G.; Kord, A. S. J. Pharm. Sci. 2011, 100, 797-812.

(30) Rozet, E.; Ziemons, E.; Marini, R. D.; Boulanger, B.; Hubert, P. Anal. Chem. 2012, 84, 106-112.

(31) Parr, M. K.; Schmidt, A. H. J. Pharm. Biomed. Anal. 2018, 147, 506-517.

(32) Berkowitz, S. A.; Engen, J. R.; Mazzeo, J. R.; Jones, G. B. Nat. Rev. Drug Discovery 2012, 11, 527.

(33) De Bièvre, P. Accredit. Qual. Assur. 2012, 17, 231-232.

(34) Olive, D. J. In Statistical Theory and Inference; Olive, D. J., Ed.; Springer International Publishing: Cham, 2014; pp 29-79.

(35) Mandel, L.; Sudarshan, E. C. G.; Wolf, E. Proc. Phys. Soc., London 1964, 84, 435.

(36) Buckingham, E. Phys. Rev. 1914, 4, 345-376.

(37) Sharma, U.; Gleason, N. J.; Carbeck, J. D. Anal. Chem. 2005, $77,806-813$.

(38) Likhachev, E. R. Tech. Phys. 2003, 48, 514-515.

(39) Huber, M. L.; Perkins, R. A.; Laesecke, A.; Friend, D. G.; Sengers, J. V.; Assael, M. J.; Metaxa, I. N.; Vogel, E.; Mareš, R.; Miyagawa, K. J. Phys. Chem. Ref. Data 2009, 38, 101-125.

(40) Squire, P. G.; Moser, P.; O’Konski, C. T. Biochemistry 1968, 7, $4261-4272$.

(41) González Flecha, F. L.; Levi, V. Biochem. Mol. Biol. Educ. 2003, 31, 319-322.

(42) Jachimska, B.; Wasilewska, M.; Adamczyk, Z. Langmuir 2008, $24,6866-6872$.

(43) Erickson, H. P. Biol. Proced. Online 2009, 11, 32.

(44) Hawe, A.; Hulse, W. L.; Jiskoot, W.; Forbes, R. T. Pharm. Res. 2011, 28, 2302-2310.
(45) Yusko, E. C.; Bruhn, B. R.; Eggenberger, O. M.; Houghtaling, J.; Rollings, R. C.; Walsh, N. C.; Nandivada, S.; Pindrus, M.; Hall, A. R.; Sept, D.; Li, J.; Kalonia, D. S.; Mayer, M. Nat. Nanotechnol. 2017, 12,360 .

(46) Cottet, H.; Martin, M.; Papillaud, A.; Souaïd, E.; Collet, H.; Commeyras, A. Biomacromolecules 2007, 8, 3235-3243.

(47) Chamieh, J.; Cottet, H. Journal of Chromatography A 2012, $1241,123-127$. 